\title{
123. Research on realization of force feedback of vehicle remote control station steering simulation system
}

\author{
Shuyuan Xing ${ }^{1}$, Tao Jiang ${ }^{2}$ \\ Changchun University of Science and Technology, Changchun, China \\ ${ }^{1}$ Corresponding author \\ E-mail: ${ }^{1 x i n g s y 5296 @ 163 . c o m, 2 j i a n g t a o @ c u s t . e d u . c n ~}$
}

Received 25 June 2017; accepted 26 June 2017

DOI https://doi.org/10.21595/jme.2017.18798

Check for updates

\begin{abstract}
Automotive steering system is able to directly experience the road information, directly determine whether the car is in a stable state of device, good fast steering system directly determine the stand or fall of the console, so the steering force feedback also gradually become the research hot spot. This paper mainly through torque motor as the main driver of steering system components, through the central processing unit of remote feedback torque is analyzed and the driver, and then send the command control and reversing and the size of the output torque of the motor to achieve steering and correction of force feedback.
\end{abstract}

Keywords: remote control, steering system, force feedback, data processing.

\section{Introduction}

Now for teleoperation car steering simulation quality directly decides the stability and safety of manipulation, the steering wheel force feedback simulation effect is good or bad will directly affect the pilot immersive in the sense of touch, the last is directly reflected in the accuracy of remote vehicle handling and so force feedback to realize remote control vehicle steering simulation system problem research mainly embodies in three aspects [1], one is for automotive steering system modeling can reflect after being accused of real vehicle test torque feedback model, and the second is for remote accused the vehicle back to the real time data processing, the third is to force feedback component torque motor control strategy research, through the pilot and the controlled object of real time data fusion processing and calculation of the control algorithm, eventually produce reasonable expectation torque output, to achieve the closed-loop stability of the "people-car-environment", meet the drivers of the controlled object accurate operation and perfect immersive, thereby reducing the driver's fatigue [2].

\section{Remote control platform steering system structure and modeling}

Force feedback of steering simulation system block diagram is shown in Fig. 1 [3], mainly by the control system based on the pilot operation information (steering wheel Angle, torque) collection, converted to digital signal is sent to $\mathrm{PC}, \mathrm{PC}$ will receive the information and data information of the controlled system (speed, acceleration and attitude parameters, road conditions, etc.), the control system through certain control algorithm, draw close to the real road driving torque, sending control command, control actuators for the torque output, make the driver to get the corresponding force.

Steering force feedback is motion information about cars and wheel surface complex of information feedback, the higher the strength of the force feedback, information, the motion of vehicle drivers on the road to grasp the more accurate, more precise control of the remote is accused of vehicles, if the force feedback is too strong, easy to make the driver fatigue, reduce system manipulation comfort, so in order to better able to give the driver force feedback [4]. The modeling of the system takes into account the stiffness, friction and other parameters of the steering torque and the steering system.

Due to the force of the steering wheel, the force mainly comes from the interaction between the ground and the steering wheel and the friction between the system itself and the gravity caused 
by the internal displacement of the main pin.

The relationship between the positive torque and the lateral force of the vehicle tyre, please refer to Eq. (1):

$T_{1}=F_{y}\left(l_{t p}+l_{h q}\right)$.

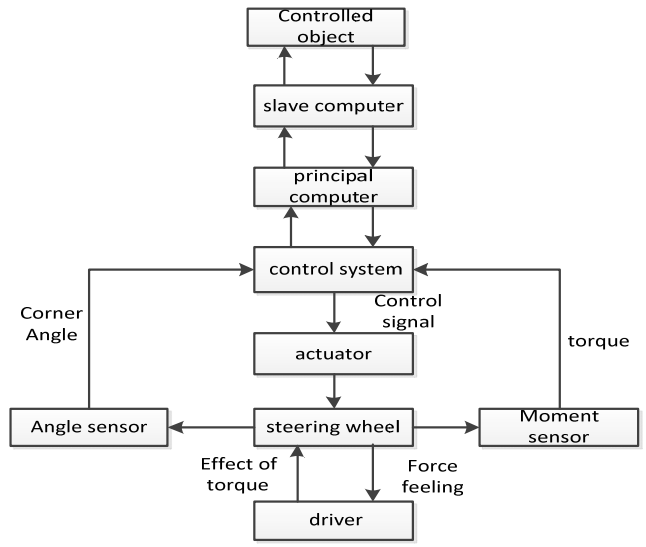

Fig. 1. The force feedback block diagram of the steering simulation system

In the course of driving, the vehicle generally considers the lateral force to be linearly dependent with the side Angle, please refer to Eq. (2):

$F_{y}=-C \alpha$.

The positive torque produced by the main pin is independent of the speed of the car, its equation please refer to Eq. (3):

$T_{2}=\frac{Q \times l_{n q}}{2} \sin 2 \theta \sin \delta$.

The vehicle tire is returning the positive moment, its equation please refer to Eq. (4):

$T_{w}=T_{1}+T_{2}=-C \alpha\left(l_{t p}+l_{h q}\right)+k \delta$.

Due to the consideration of the forward direction, the feedback of the road is only related to the forward torque of the front tire, and the front wheel Angle of the car, its equation please refer to Eq. (5):

$\alpha_{f}=\beta+\frac{l_{f} \gamma}{v}-\delta$.

The force please refer to Eq. (6):

$F_{f l}=F_{f r}=-C_{f}\left(\beta+\frac{l_{f} \gamma}{v}-\delta\right)$.

Therefore, the torque of the front wheel is convert to the torque please refer to Eq. (7):

$T_{z}=-\frac{2 C_{f}\left(l_{t p}+l_{h q}\right)}{N_{p w}} \beta-\frac{2 l_{f} C_{f}\left(l_{t p}+l_{h q}\right)}{N_{p w} v} \gamma+\frac{2 C_{f}\left(l_{t p}+l_{h q}\right)+\left(Q \times l_{n q}\right) \sin 2 \theta}{N_{p w}} \delta$. 
Modeling of steering resistance moment can clearly found that if you want to get steering resisting moment, just need to know the car swing angular velocity, Angle, total want to acceleration, and vehicle side-slip Angle, and these values are through the vehicle velocity, velocity and Angle sensor to measure directly, at the same time, car side-slip Angle formula is as follows: $\beta=\tan ^{-1}\left(v_{y} / v\right)$, By controlling the dc torque motor through the above data, the torque output of the dc torque motor is realized, and the operation stability and accuracy of the system are achieved.

\section{Data transmission and processing}

In the process of remote control, the greater the transparency of the system, the better the operating sense of the system, the better the driver can be immersed in the driving process.

In order to make the system more transparent, the most important problem is the delay of the system. The delay of the system is always a problem in the process of remote operation.

At present most of them are based on $3 \mathrm{G}$ or $4 \mathrm{G}$ network transmission [5], due to its transmission depend on the data transmission between the base station and base station, and the transmission speed is limited by the same base station at the same time using the number, if a base station at the same time there are a lot of people in the download data, so everyone's transmission rate will drop, so the transmission speed of great uncertainty, so this article chooses is wireless digital radio, whose system diagram below.

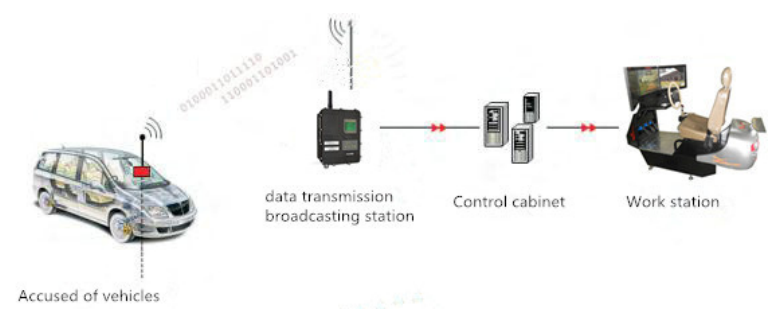

Fig. 2. System simplified schematic diagram

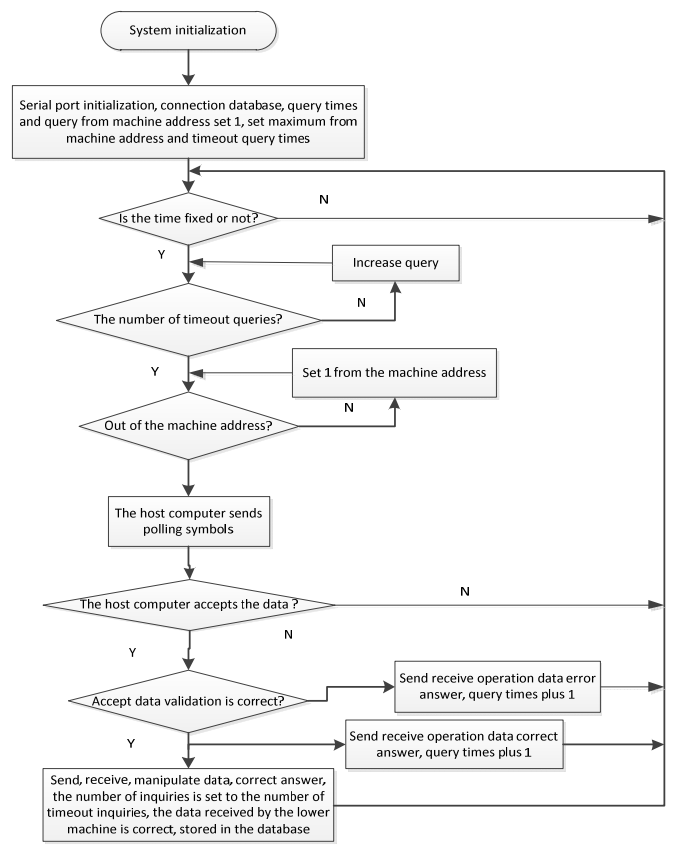

Fig. 3. The data transmission flow of the remote operating system using radio digital radio 
First is the remote control vehicle's motions and gestures transferred to $\mathrm{PC}$, digital radio transmission time is $10 \mathrm{~ms}$ [6], each has its own digital radio spectrum, was occupied by other people don't have to worry about data and lead to the uncertainty of data transmission delay, through the control system for data analysis and processing, to control the rotation and the size of the output torque of motor, force sensing feedback to the driver, at the same time, the system will be the operator of data information transmission distance is accused of vehicles, accused by the actuator to realize the vehicle.

Isn't all of the data in the process of data transmission of all accepted, but selectively accept after analyze the data, if the accused car suddenly hit the rocks or in ravines, its data transmission data will suddenly change, there will be no rules to follow, this time should be after the data processing for feedback to the driver, at the same time, combining with the video data, which can ensure that reduce the discomfort of the pilot operation, also can ensure the driver. The data transmission flow of the remote operating system using radio digital radio is as follows.

\section{Research on force feedback control strategy of remote control console Steering simulation system}

In the car remote control steering simulation system in Taiwan, is relying on rotating DC torque motor driven rotating the steering wheel, to provide information and sense of the way back to the steering wheel drive, giving the driver force feedback, so the performance of the whole station of the steering force feedback plays a decisive role in the performance of the steering system, so the control strategy is also is the control strategy of motor research. In order to realize the rapid response of the system and enhance the transparency of the system, the 70LYX07 rare earth permanent magnet DC torque motor with low speed and high torque is adopted.

The traditional PID control is used to ensure that the operator can feel soft and satisfy the system's torque.

Traditional PID control is mainly composed of controller and the controlled object, the PID controller signal is according to the given value $r(t)$ and the output value $y(t)$ for the control deviation value, at the same time of deviation proportional, integral, differential operation, then to control the controlled object, its output $u(t)$ algorithm for [7]:

$u(t)=K_{P}\left[e(t)+\frac{1}{T_{i}} \int e(t) d t+T_{d} \frac{d e(t)}{d t}\right]$.

According to different to the three parameters of pid control system, the performance of the proportional coefficient $K_{P}$, directly determines the strength of the control, increasing the proportion coefficient can enhance the system's dynamic response speed, but if too much, and will cause damage to the stability of the system, and may cause the system unstable; The integral part is mainly to improve the error of the system, so that the system can eliminate the steady-state error, but it will increase the reaction time of the system and slow the response rate. Differential link can improve system stability and response speed at the same time, the system error can foresee, produce lead correction, reduce the overshoot, enhance the stability of the system, the shortage of the is in enhance the system performance index is also amplified noise signal, so in the practical engineering, usually according to the requirements of system control mode selection.

The parameter setting of PID is determined based on the empirical formula and the combination of the test and determination. Firstly, the parameters of the current loop are determined and simulated, and the selection of $P=0.5, I=60$, its step response curve is shown in Fig. 4.

The step response curve can be seen that the system is not overregulated, and the stable state can be achieved at $0.5 \mathrm{~s}$, which can realize the rapid change of current and ensure the precision of the driver's control.

For the parameter setting of speed ring, select $P=1$, the step response curve of $I=5$ and the 
following of the system are shown in Figs. 5, 6.

The curve shows that the step response of the system can meet the system requirements, and the system is very good with the following, and can provide good feedback for the operation of the driver.

Of current and speed PI control can enhance the quickness of motor start, at the same time guarantee the smoothness of the motor start, guarantee the stability of the motor current and rotating speed, avoid caused by current instantaneous changes of system instability and interference on the system, to ensure the torque changes gentleness and follow performance of the system, the changes in the size of the force can quickly enough accurate feedback from the pilot, in operation at the same time feel more moderate, enables the process of pilot control good experience the speed of the car and car body posture change, the control of the remote car is also more accurate, more comfortable experience.

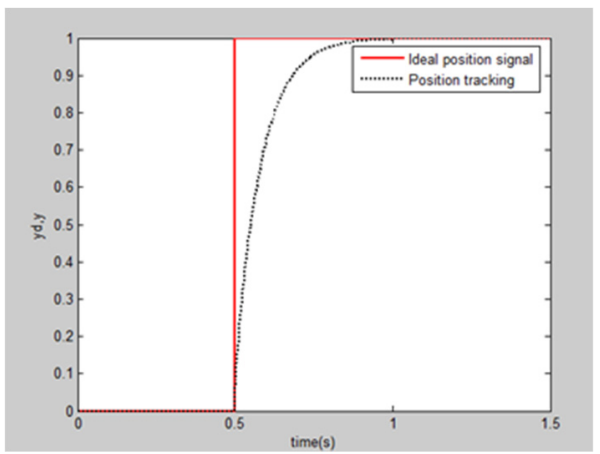

Fig. 4. Step response curve of current loop PID control

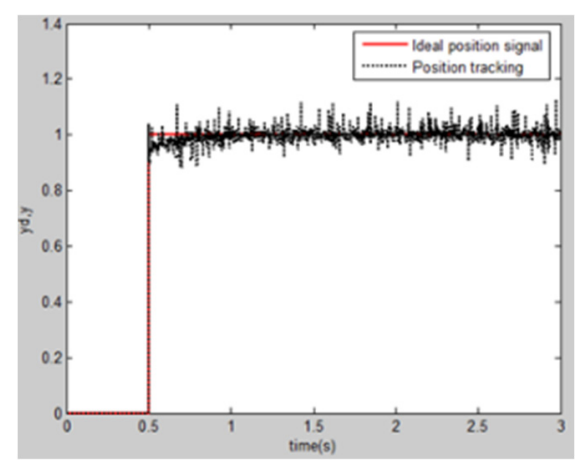

Fig. 5. Step response curve of speed loop PID control

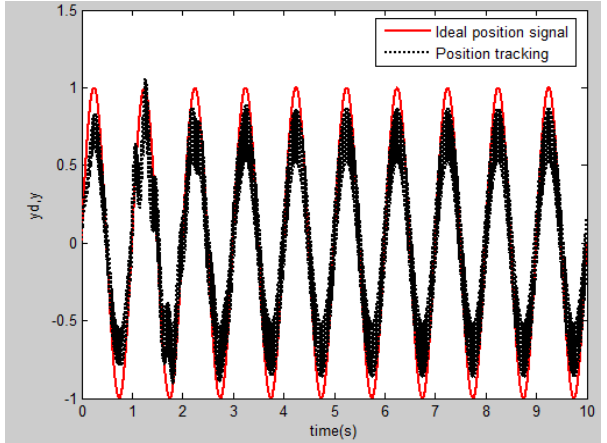

Fig. 6. System following curve

\section{Conclusions}

With force feedback effect of the steering system dynamics modeling analysis, put forward the control strategy of system, through the control of dc torque motor and reversing, and the size of the torque output, make the operator feel force, and through to the dc torque motor control algorithm design, make the dc torque motor current and torque has good tracking performance, for excessive deviation corrected in time, reduce the influence of interference on the system, and enhance the operators in the process of manipulating soft, lightweight and real road feel, to avoid the big impact, and also can reduce in the process of manipulation of the remote car is not allowed to grasp the situation, can according to the torque motor, the force feedback to adjust the speed of the car, let the driver accomplish know fairly well, in order to achieve in the real environment away from the car to car's running state and gestures of the precision, is remote control car safety driving. 


\section{References}

[1] Zheng Hang Research and Implementation of Steering Force Feedback of Driving Simulator. Jilin University, 2016.

[2] Zhao Wei Simulation of the Loop System Based on the Steering of the Steering System of the Steering System. Hunan University, 2012.

[3] Huang Huo The Research and Development of Vehicle Driving Simulator to Simulation System. Zhejiang University, 2012.

[4] Chen Yang Research on the Feedback Control Technology of Linear Control Steering Force. Harbin Institute of Technology, 2010.

[5] Kwon J., Park K. Development of the hardware-in-the loop simulator for evaluating performance of EPS system. Asia Pacific Automotive Engineering Conference, 2011.

[6] Liu Weiguo Application and design of wireless digital radio in remote monitoring system. Mining Machinery, Vol. 8, 2008, p. 44-46.

[7] Aström K. J., Hägglund T. PID Controllers: Theory, Design and Tuning. Instrument Society of America Research Triangle Park Nc, 1995. 